\title{
Sensitivity analysis in connectionist modeling
}

\author{
WALTER SCHNEIDER \\ University of Pittsburgh, Pittsburgh, Pennsylvania
}

\begin{abstract}
The application of sensitivity analysis methodology to connectionist modeling is discussed. Sensitivity analysis examines the functional relationship of parameters in dynamic systems to specify the interactions of parameters and the boundary conditions of dynamic system models. Sensitivity analysis is contrasted with parameter estimation. Reasons to favor sensitivity analysis in connectionist modeling are discussed. Six steps for sensitivity analysis are detailed with examples from the modeling of short- and long-term memory retrieval.
\end{abstract}

When modeling phenomena in psychology, one tries both to understand the interactions in the model and to compare the model's performance with some empirical database. Connectionist modeling provides a new approach (see Rumelhart \& McClelland, 1986; Schneider, 1987), which places emphasis on alternative techniques for assessing models. This paper does not deal directly with specific connectionist modeling procedures. For information on specific models, the reader should refer to McClelland and Rumelhart (1988a, 1988b; Rumelhart \& McClelland, 1986) and Munro and Anderson (1988). The focus in this paper is on how one analyzes the behavior of any model in order to understand its mechanisms and to compare the model's performance with human data.

Historically, most modeling in psychology has been of the parameter estimation type (e.g., Atkinson, Bower, \& Crothers, 1965; Wickens, 1982). Parameter estimation involves finding the single best set of parameters to enable comparing summary statistics between a model and the observed empirical data. Such parameter estimation is important for evaluating multiple models for a given phenomenon. There are well-established techniques for doing efficient parameter searches in multidimensional spaces to determine the best-fitting function (e.g., Press, Flannery, Teukolsky, \& Vetterling, 1986).

Unfortunately, parameter estimation provides little information about the interactions within a model. Typically, a computer program performs a grid search, evaluating the model's fit for many points in a parameter space. The final result is the set of parameters that give the best fit. Students can obtain the best-fitting results without ever knowing how various parameters in the model trade off or even whether their model has been correctly programmed.

Sensitivity analysis provides more information than does parameter estimation on the behavior of a model. Sensitivity analysis draws out functional relationships, report-

This research was sponsored by Office of Naval Research Contracts N00014-87-K-0397 and N00014-86-K-0678 and Army Research Institute Contract MDA903-86-C-0149. Reprint requests can be sent to Walter Schneider, LRDC Building, 3939 O'Hara St., University of Pittsburgh, Pittsburgh, PA 15260. ing the behavior of the system as a function of the parameters varied over the boundary conditions in which the system should operate. It provides an assessment of trade-offs to find what combinations of parameters are best in which situations. Reporting a sensitivity analysis requires functionally describing the interactions within the model.

Sensitivity analysis was developed in the engineering discipline of systems analysis of dynamic systems (see Cruz, 1973; Tomovic, 1963). Systems analysis examines the behavior of complex systems such as control systems and aircraft flight dynamics. Engineers do sensitivity analyses typically to determine trade-off points in a design or to increase system reliability to component variability. There is no "best" wing design; rather, one can trade off factors such as maneuverability, lift, altitude range, and speed for performance of the system in flying various missions. Sensitivity analysis is also important in determining the reliability of a system. For example, in engineering electronic systems (see Mitra, 1969; Truxal, 1972), the designer seeks to build a system that will work equivalently when the components used to assemble the system have substantial variation (e.g., the resistors used have a $10 \%$ variation around the specified value).

Sensitivity analysis is important to the connectionist modeler because a modeler should specify the boundary conditions of any model, the sensitivity of the model to any parameter change, and the trade-offs that occur between parameters in the model. Before a modeler discusses a parameter at length, it is important to demonstrate that the parameter has an impact on the performance of the model, and that the model is robust to reasonable variation of the parameter. It is also important to determine the boundary conditions of the other parameters over which the variable of interest influences system performance.

Sensitivity analysis identifies the interactions of variations of parameters to determine where changes in components have a large impact on the system's performance. Often, later stages turn out to be quite insensitive to variations of internal parameters. For example, if one stage increases its output but feeds that output to another stage that is near saturation, there is no impact of the change. 
Sensitivity analysis also determines situations in which the system should allow variation of the parameters rather than fix a parameter. The swept-wing design of modern fighter aircraft illustrates the design of a system that allows a range of values of a parameter. At low speeds the wing is fully extended to maximize lift, whereas at high speeds it is swept back at an angle to reduce drag and lift. There is no "best" wing placement. In fact, the system's performance varies so dramatically that it is best to have a range of wings by sweeping the wing back at various angles. Biological systems use parameter variation (e.g., blood temperature during fever) to vary the dynamics of the system. Doing a sensitivity analysis encourages the modeler to ask, "what if the system could do both A and B," whereas parameter estimation ends with "which is better: A or B."

Sensitivity analysis is generally defined as the percent change in the output $(Y)$ of a system as a function of a percent change in a system parameter $(X)$ (see Tomovic, 1963 , pp. $25-30$, or Truxal, 1972, p. 403):

$$
S_{X}=\frac{d Y / Y}{d X / X} .
$$

In other words,

$$
\text { Sensitivity of } Y \text { to } X=\frac{\% \text { change in } Y}{\% \text { change in } X} \text {. }
$$

The sensitivity is generally calculated with small changes of the parameters of the system. ${ }^{1}$ For example, we could measure the change in simulated recall from a model to a change in the learning rate of the model for a given set of parameters. If the the recall improves by $0.5 \%$ to a change in learning rate of $1 \%$, the sensitivity of the model would be 0.5 . If the model is robust to a given parameter change, one would expect to see sensitivities substantially below 1 .

The interpretation of a parameter is dependent on the sensitivity of that parameter and the other parameters of the model. If the sensitivity of one parameter is very large compared with all the rest of the parameters, one can discuss that parameter as having the major controlling influence on the model. If the performance is insensitive to the value of the parameter, then it is inappropriate to attribute the behavior of the system to the value of that parameter. If several parameters are sensitive, one must be cautious to detail the interactions among the parameters.

There are seven aspects of connectionist modeling that argue for the importance of doing sensitivity analysis. First, modern connectionist modeling is still in its infancy (it is less than 10 years old; see Schneider, 1987), and we are just beginning to explore the range of models. There are many potential arrangements of units and levels, types of learning rules, and regimes for training. In this early stage of exploration, it is better to ask how does the system's behavior change with various assumptions rather than what is the best single set of assumptions.

Second, connectionist modeling typically involves varying many free parameters with powerful learning rules, which can produce fits to any data set or even fit a buggy model to the data. Massaro (in press) illustrated how a single connectionist model can fit human acoustic data or equally well fit data never observed in humans. Debugging a connectionist model can be particularly problematic. A programmer may have to transform a complex equation in order to implement it for fast computer simulation. It is difficult to debug a model that is running and providing reasonable fits. I once used a connectionist model with a serious bug in it for months, getting good fits, before I realized there was something wrong. I found the error during a sensitivity analysis by running the simulation at a boundary condition of one of the parameters and observing unreasonable behavior from the system.

Third, connectionist models are full of nonlinearities (e.g., a logistic function transformation of activation). Such nonlinearities can reduce the impact of variations in parameters. Without doing a sensitivity analysis, the modeler is unlikely to determine whether a parameter has any impact on performance.

Fourth, all connectionist models are at best simplistic approximations to the system being modeled. For example, most human cognition probably requires the interactions of at least tens of thousands of units, and probably millions. Hence, whether a model fits with a hundred or a thousand units is of little importance. It is, however, important to show that the model still performs well as the number of units increases, so that it is conceivable for the model to work if it is scaled up to human proportion.

Fifth, it is difficult to directly compare alternative connectionist models on the basis of fit. For example, added parameters in one model may not affect performance, so that two models described very differently can be effectively the same model.

Sixth, it is important to communicate to colleagues the important parameters and their interaction. Before an author presents an extended discussion of the importance of a parameter, it is his/her responsibility to communicate the sensitivity of the system to that parameter. We have scientific conventions that one cannot discuss a difference between conditions that is not statistically reliable (e.g., with a $p<.05$ ). Similarly, one should not discuss a parameter until one can demonstrate that variations in the parameter substantially alter the behavior of the system.

Seventh, and most important, modelers have difficulty understanding the behavior of their own connectionist models, and sensitivity analysis can reduce some of the uncertainty. A standard question I ask new student modelers is, "What will the system do if you double each parameter individually?"' Most novice connectionist 
modelers cannot provide even qualitative estimates of such changes with certainty. By doing the sensitivity analysis, students gain an appreciation for what matters in the model, how assumptions trade off, and what the boundary conditions of the model are.

Doing a sensitivity analysis can produce better models. A sensitivity analysis helps a researcher understand a model, indicates the critical aspects of the model, shows the robustness of the predictions, and identifies boundary conditions of the model. It also allows the researcher to better debug the model.

\section{Performing a Sensitivity Analysis}

Connectionist modeling can be a difficult challenge to the researcher, sometimes consuming the researcher's resources and patience. In studies involving learning in large networks, training the system to acquire a large ensemble of patterns can require days of computer time. Hence fine-grain grid searches of parameters are often not feasible. Also, there can be large numbers of parameters, and it is difficult to keep track of how one variable interacts with all the other parameters in the system.

Based on my experience in connectionist modeling (e.g., Schneider \& Detweiler, 1987) and more traditional mathematical modeling (e.g., Shiffrin \& Schneider, 1977), I suggest some guidelines for sensitivity analysis to the prospective modeler. These are neither necessary nor sufficient guidelines for successful modeling, but rather suggestions for steps that are likely to make the modeling effort more fruitful.

I illustrate six steps with examples from a connectionist model of working memory (Schneider \& Detweiler, 1987). The model was applied to modeling short-term memory retention. For the present purposes, I describe the analysis of two parameters of the model: the learning rate and the degree of correlation between retrieval cues. The correlation modeled the effect of context similarity across learning trial presentations. We assumed that there was some internal context vector and that elements of the vector changed randomly over time. In the sensitivity analysis, we examined the trade-off between the learning rate and the degree of correlation between retrieval cues. We examined both immediate recall, as in a short-term memory experiment, and delayed recall, as in end-ofsession recall.

1. Lay out the set of possible parameters and reasonable ranges. Identify for each parameter what the reasonable range is and guess what the best value of the parameter would be for the model. For example, the reasonable learning constant for a model (i.e., the amount to change the strength of connections after a learning trial) could range from 0 (no learning) to 1.0 (perfect learning of each pattern on a single trial). Based on past experience and the published literature, one can guess what level would be best for the current task. In pattern association learning, a typical learning rate per pattern is 0.1 . Next, one should specify the range for each of the parameters.
In the working memory model, a second parameter was the correlation between input retrieval cues, which could range from -1 to 1 . However, it seems unlikely that naturally occurring contexts could produce negative correlations, so we examined sensitivity in the range of 0 to $1.0^{2}$ For events that were expected to occur in close time periods (e.g., within $30 \mathrm{sec}$ ), we expected the correlation to be very high $(0.9)$ and to decay exponentially with time. We set the vector size to 200 elements and generated random vectors so the results would provide good estimates of the performance of the system with long vectors. $^{3}$

2. Individually set parameters to minimum and maximum range to verify expected behavior. To save time, initial tests should be carried out with simple test problems and then with the full problem. This is critical for debugging a model and beginning to understand the behavior of the system. To minimize computer time, it is often best to work with small networks and small ensembles. For example, one might want to model a network with 100 units, but run tests with 5,15 , and 100 units. Desk check single presentations of the smallest-sized networks to verify that the model numbers match the numbers resulting from the mathematical analysis. With the middle-sized networks, set the parameters individually to the boundary conditions and verify the expected behavior. This involves setting all the parameters to their expected values and then sequentially taking each parameter and running the simulation with that parameter at the lower boundary, expected best value, and maximum boundary. For example, in a pattern association network without hidden units (see McClelland \& Rumelhart, 1988a), setting the learning rate to 0 should cause no change in performance over trials, whereas setting the rate to 1.0 should cause perfect learning of the network, such that immediately after learning the network the output for a given input can be reproduced and there is no change in the network with repeated presentations of the same pattern. A common error in connectionist modeling is to miscalculate the effective learning rate, causing too high a learning rate. Such an error causes oscillation as the network overshoots the desired association between trials. If the learning rate is less than 2.0 , the model may still show gradual improvement over trials, even though it overshoots on every trial. Checking the boundary condition provides an easy global test of the mathematical behavior of the model.

3. Plot out the behavior of the system with a coarse grid of points for the individual parameters. I suggest a grid of five points: the minimum and maximum boundary conditions, the expected best values, and the points halfway between the best value and the boundary conditions. The researcher should choose specific points based on where major changes in the performance of the model are expected. If there are four parameters with five points each, the simulation model would have to be run 20 times. In the working memory model, the parameters were the learning rate, degree of correlation, number of units, and 
number of learning trials. The performance of the model was quite sensitive to the learning rate and degree of correlation. The number of units had little effect above 50 units, and the number of trials had a large effect between the first and second trial, but small effects beyond the fourth trial. A model's performance should be examined using a variety of metrics. In testing the working memory model, we examined recall after each trial and at the end of the session. We used multiple recall metrics, including measures of the correlation, number of units matching, and best-guess vector.

After the simulation data are collected, the results should be plotted to determine the qualitative behavior of the system in the dimensional space of the parameters. I usually examine two graphical relationships, plotted by an empirical graph and a sensitivity graph. The empirical graph plots the data on axes that are most representative of the human data that are being simulated (see Figure 1). To keep the graphs from being too cluttered, one can plot the data for only a few of the dimensions on a single graph. For the working memory experiments, we plotted recall as a function of trials, with separate lines for each combination of learning rate and correlation between vectors. This type of graph illustrates the data in a way that is most natural for empirical researchers in the field.

\section{Learning Rate}

\section{A Learning}

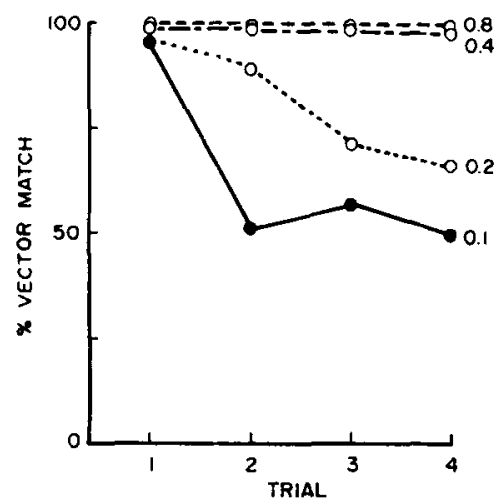

\section{B Retention}

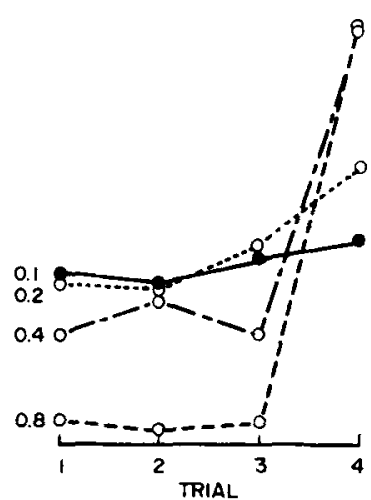

Retrieval Correlation

C Learning

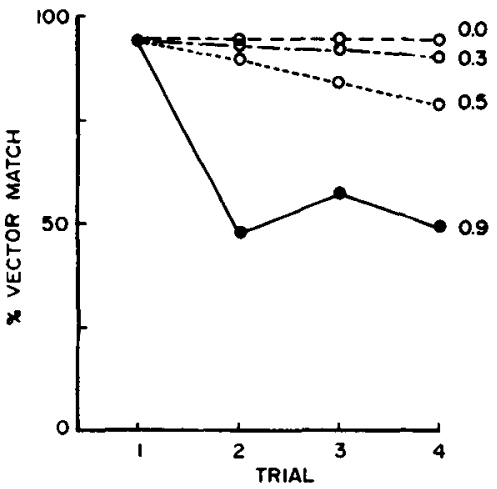

D Retention

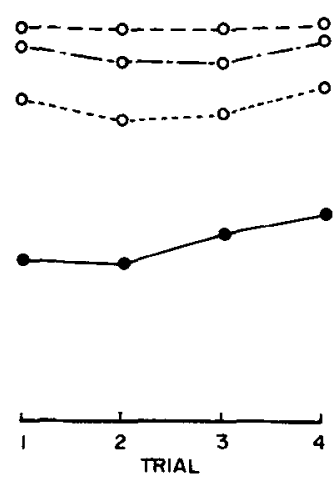

Figure 1. Simulated recall data for a connectionist model as a function of the learning rate $(A, B)$ and correlation between retrieval cues $(C, D)$. The left figures show recall at the end of the trial, as would be representative of a short-term memory experiment. The right figures show recall of all the patterns after all four have been learned. For panels $A$ and $B$, the retrieval correlation was set to 0.9 and the learning rate was varied with the values shown in the figure. For panels $C$ and $D$, the learning rate was set to 0.1 and the vector correlation was varied. (Adapted from Figures 6 and 8, pp. 86 and 97, in "A Connectionist/ Control Architecture for Working Memory" by W. Schneider and M. Detweiler, in The Psychology of Learning and Motivation, Volume 21, by G. H. Bower, Ed., 1987, New York: Academic Press. Copyright 1987 by Academic Press. Adapted by permission.) 
Figure 1 illustrates a set of empirical graphs derived from the working memory model (Schneider \& Detweiler, 1987). The left-hand graphs are plotted in a manner typical of short-term memory experiments. The right-hand graphs show end-of-session recall as would occur in a free recall experiment. There are separate lines for each of the parameter values used in the grid search. The upper graphs show the effects of the learning rate parameter, and the lower graphs illustrate the effect of correlation of the retrieval vectors. Visual inspection of the graph suggests that clear qualitative differences occur. For example, on Trial 3, comparing a high learning rate (0.8) to a low learning rate $(0.1)$ produces good immediate recall (Figure 1A) but very poor end-of-session recall. However, these differences are not present when the correlation between retrieval cues is reduced (see Figures $1 \mathrm{C}$ and $1 \mathrm{D}, 0.3$ correlation condition).

The sensitivity plots illustrate the sensitivity of the model to variations of the parameters, highlighting the causal influence of various parameters on the performance of the model. By plotting the data as a function of the parameters, one can see the qualitative behavior of the model. From the graphs, one can determine whether the model is sensitive to changes of the parameter and how the parameters interact.

Figure 2 illustrates sensitivity plots of the same data shown in the empirical plots in Figure 1. The qualitative effects of the parameters are more evident in Figure 2 than in Figure 1. Several qualitative effects are illustrated. First, for Trial 1 the model's immediate recall performance (Figure 2A, top dotted line) is uninfluenced by the learning rate above 0.1 , whereas for Trial 3 (Figure 2A, top solid line) it increases until saturation. ${ }^{4}$ Increasing the learning rate improves immediate recall at the expense of decreasing end-of-session recall (Figure 2A, solid lines). There is no optimal learning rate that gives good immediate and delayed recall. Figure 2B illustrates that a difference between Trial 1 and Trial 3 recall occurs only when there is a high correlation between vectors (i.e., above 0.3 ) and not when there is a low correlation. The

A

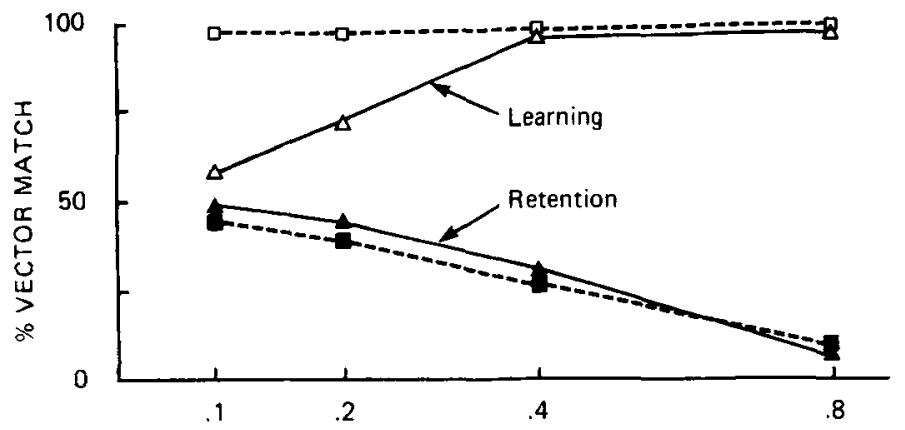

LEARNING RATE

B

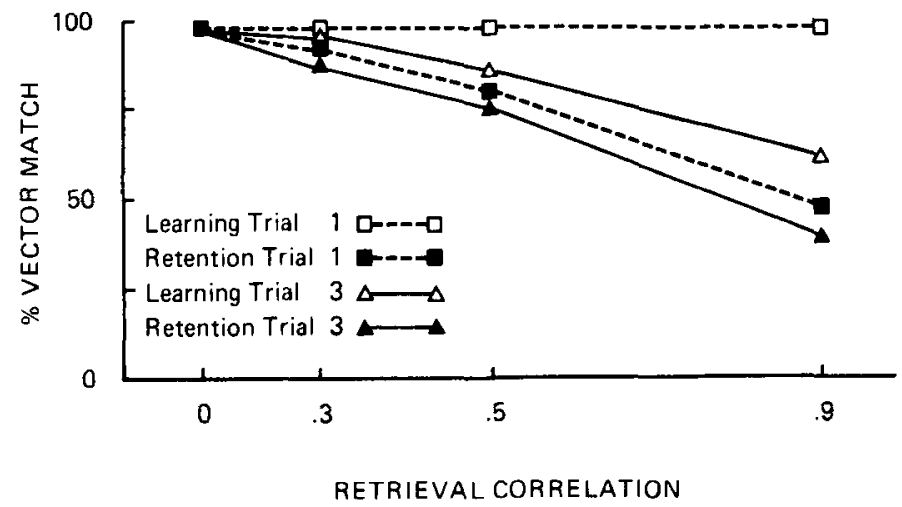

Figure 2. Sensitivity functions showing recall performance as a function of the parameters. Panel A varies learning rate while holding vector correlation constant at 0.9. Panel B varies retrieval correlation between successive vectors while holding the learning rate constant at 0.1 . (This figure plots the data shown in Figure 1 on model parameter axes.) 
interaction between the learning rate and the correlation indicates the boundary conditions over which learning performance differs between Trial 1 and Trial 3.

4. Examine the effects of each variable to identify sensitive regions of the model and to determine the bifurcation values for each parameter. The sensitive regions are the ranges of the parameter values over which the performance of the model is sensitive to changes in the parameters (e.g., where the sensitivity values are above $0.1)$. The bifurcation points are the values of the parameters at which the behavior of the model changes qualitatively. In engineering, the bifurcation points are operationally defined as the points at which a linear model of the parameters can no longer adequately predict the behaviors of the system and new terms or equations must be introduced (see Tomovic, 1963). Varying parameters in a connectionist model often produces a nonlinear effect on performance. Many parameters exhibit a sigmoid shape with three regions of performance. The first region is a prethreshold region, where there is little change until a threshold is reached. The second region is the sensitive region, where performance changes monotonically with increasing values of the parameter and can often be fitted with a linear function. The third region, the saturation region, is where changes in the parameter have little effect on performance. The whole set of graphs should be examined to determine where the bifurcation points are and whether the locations of these points vary as a function of other parameters.

The number of units used in a hidden layer of a connectionist model illustrates the saturation effect. Learning generally improves as the network has more hidden units between the input and output units. However, there is a diminishing marginal utility of adding units, so that beyond a certain point adding more units has little impact on the performance of the model. Sejnowski and Rosenberg's (1987) analysis of NET-TALK illustrates a saturation effect. NET-TALK learns to associate letter strings to phonemes, enabling the model to accurately specify the phonemes for written text. The association between input and output occurs in a layer of 0 to 120 units connecting the text input and the phonemic output. As the number of hidden units increases, performance of the model increases. After 30,000 learning trials, the model's best-guess accuracies with $0,15,30,60$, and 120 hidden units were $82 \%, 88 \%, 92 \%, 94 \%$, and $95 \%$, respectively. The model becomes insensitive to changes in the number of hidden units between 30 and 120 units, where adding more units has little impact on performance.

5. Examine the interactions of the parameters and the types of tests. In the working memory model, Schneider and Detweiler (1987) examined how the learning rate influenced the immediate and end-of-session recall and how performance changed as a function of learning trial (see Figures 1 and 2). Several interesting interactions appeared. First, there was no effect of learning rate for im- mediate recall on the first trial (Figure 2A, Trial 1). Learning for Trials 2-4 increased with increases in the learning rate from 0.1 to 0.4 , reaching saturation above 0.4 . However, the data showed a dramatically different pattern when the retention data rather than the end-ofsession recall data were considered. For Trials $1-3$, the learning and retention data were nearly reversed. ${ }^{5}$ Larger learning rates (from 0.1 to 0.8 ) led to better end-of-trial learning and worse retention (Figure 2A).

The results in Figures 1 and 2 illustrate how sensitivity analysis can be more informative than merely finding the single best parameter fit. What is best depends on the situation. If the task requires rapid learning without later retention, learning rates above 0.4 are best. If the goal is retention, learning rates around 0.1 are best. As a result of these trade-offs, an optimal system may incorporate both large and small learning rates, giving both rapid learning and reasonable retention. We proposed such a system, and the human behavioral data (see Schneider \& Detweiler, 1987) and the animal physiological data (see Mishkin, Malamut, \& Bachevalier, 1984) suggest the existence of multiple learning rate constants. The sensitivity analysis identifies trade-offs that suggest where a biological system might develop a hybrid system to produce good performance on a wide range of points.

6. Report the sensitivity analysis in such a way as to give the reader an appreciation for the boundary conditions and interactions of the parameters. In a paper describing a sensitivity analysis, the author's goal is to communicate the functional relationships in the model, not simply to describe how well the model fits the data. It is important to illustrate the behavior of the model with performance graphs showing the relationships among the parameters. The boundary conditions and bifurcation points should be discussed. The writer should specify over what range of parameters the qualitative phenomena discussed will hold. If the model operates in several subspaces of the parameters, the author should describe what parameters determine transitions from one region to the next.

\section{Conclusion}

Connectionism is a powerful modeling approach that poses new challenges to the modeling of psychological phenomena. Using powerful learning algorithms to search high-dimensional spaces often produces reasonable fits to the data. However, to make progress in exploring a class of models, we must understand how the models come to fit the data. The researcher must understand how the parameters interact and identify the boundary conditions of the model. Sensitivity analysis is a methodology developed in systems engineering to understand and predict the behavior of man-made systems. It provides methods for determining the relationships among parameters within dynamic nonlinear systems. Psychologists can adapt this methodology to better understand the models we create to predict the behavior of natural cognitive systems. 


\section{REFERENCES}

Atkinson, R. C., Bower, G. H., \& Crothers, E. J. (1965). An introduction to mathematical learning theory. New York: Wiley.

CRUz, B. J., JR. (Ed.). (1973). System sensitivity analysis. Stroudsburg, PA: Dowden, Hutchinson \& Ross.

Massaro, D. W. (in press). Some criticisms of connectionist models of human performance. Journal of Memory \& Language.

MCClelland, J. L., \& RumelharT, D. E. (1988a). Explorations in parallel distributed processing: $A$ handbook of models, programs, and exercises. Cambridge, MA: MIT Press.

McClelland, J. L., \&umelhart, D. E. (1988b). A simulation-based tutorial system for exploring parallel distributed processing. Behavior Research Methods, Instruments, \& Computers, 20, 263-275.

Mishixin, M., Malamut, B., \& Bachevalier, J. (1984). Memories and habits: Two neural systems. In G. Lynch, J. I. McGaugh, \& N. M. Weinberger (Eds.), Neurobiology of learning and memory. New York: Guilford Press.

MrTRA, S. K. (1969). Analysis and synthesis of linear active networks. New York: Wiley.

MounTCastLe, V. B. (1979). An organizing principle for cerebral function: The unit module and the distributed system. In F. O. Schmitt \& F. G. Worden (Eds.), The neurosciences (pp. 21-39). Cambridge, MA: MIT Press.

Munro, P. W., \& ANDERson, J. A. (1988). Tools for connectionist modeling: The dynamical systems methodology. Behavior Research Methods, Instruments, \& Computers, 20, 276-281.

Press, W. H., Flannery, B. P., Teukolsky, S. A., \& Vetterling, W. T. (1986). Numerical recipes: The art of scientific computing (chap. 10). New York: Cambridge University Press.

Rumelhart, D. E., \& McClelland, J. L. (Eds.). (1986). Parallel distributed processing: Explorations in the microstructure of cognition. Volume 1: Foundations. Cambridge, MA: MTT Press.

SCHNEIDER, W. (1987). Connectionism: Is it a paradigm shift for psy- chology? Behavior Research Methods, Instruments, \& Computers, 19 , 73-83.

SCHNEIDER, W., \& DETwEILER, M. (1987). A connectionist/control architecture for working memory. In G. H. Bower (Ed.), The psychology of learning and motivation, Volume 21. New York: Academic Press.

Sejnowski, T. J., \& Rosenberg, C. R. (1987). Parallel networks that learn to pronounce English text. Complex Systems, 1, 145-168.

ShIFFrIN, R. M., \& SchneIder, W. (1977). Controlled and automatic human information processing: II. Perceptual learning, automatic attending, and a general theory. Psychological Review, 84, 127-190.

Tомоvic, R. (1963). Sensitivity analysis of dynamic systems. New York: McGraw-Hill.

TrUXAL, J. Q. (1972). Introductory system engineering. New York: McGraw-Hill.

WiCKENS, T. D. (1982). Models for behavior: Stochastic processes in psychology. San Francisco, CA: W. H. Freeman.

\section{NOTES}

1. Depending on the application, the absolute value or the log of the values is used.

2. In high-dimensional spaces, it is easy to get a zero correlation but difficult to get a negative correlation, since the latter would require an exact opposite value of each of the dimensions.

3. Physiologically, a cortical macrocolumn (see Mountcastle, 1979) has an output vector of $1,000-10,000$ pyramidal cells. This suggests that a model's simulated vector operations should statistically approximate the behavior of very long vectors (e.g., a vector length of 30 or above).

4. For learning rates below 0.1 , immediate recall decreases rapidly, even on Trial 1 (not shown in Figure 1).

5. Note that Trial 4 is not a true retention point, since the recall of the last trial occurs without learning any intervening items. 